\title{
A DEEP LEARNING APPROACH FOR NOISY IMAGE CLASSIFICATION IN AUTOMATED DRIVER DROWSINESS DETECTION
}

\author{
${ }^{*}$ Marwa K. Hussein ${ }^{1}$
}

Tariq M. Salman ${ }^{1}$
Abbas H. Miry ${ }^{1}$

1) Electrical Engineering Department, College of Engineering, Mustansiriyah University, Baghdad, Iraq

\begin{abstract}
In recent years, driver drowsiness has been a major cause of road accidents, particularly when the driver has been driving on the highway for an extended period of time. Smart systems can now be used to prevent accidents, and a reliable driver detection system must be applied to alert the driver. In these systems, several external factors have been degrading the performance of these systems, including added noise, interference and low illumination. To overcome these limitations, this paper presents a de-noising approach for noisy images; the results show that the enhanced images improve the overall system performance and classification accuracy. The final validation accuracy is $97.5 \%$, while the testing accuracy for S1 is $96 \%$, S2 is $92 \%$, and S3 is $91 \%$. The test accuracy of S1 decreased to $45 \%$ when the Salt and pepper noise is added to the set , , when Gaussian noise is added to S2 the testing accuracy decreased to $85 \%$, and when speckle noise is added to S3 the testing accuracy is reduced to $73 \%$. When the median filter is used the testing accuracy for S1 become $93 \%$, the testing accuracy for S2 increase to $91 \%$, and the testing accuracy for $\$ 3$ raises to $85 \%$.
\end{abstract}

Keywords: Face detection; Drowsiness detection; Deep Learning; Noise removal.

\section{Introduction}

Traffic accidents represent one of the leading causes of death worldwide, with 1.25 million people dying each year, according to the World Health Organization (WHO) [1]. According to the National Highway Traffic Safety
Administration, drowsy driving causes thousands of deaths and accidents each year in the United States. A drowsy driver is involved in $10 \%$ of fatal daytime accidents, $24 \%$ of fatal nighttime accidents, and $15 \%$ of all fatal accidents in the United States [2]. According to this estimation, nearly 6000 people died in drowsy-driving car crashes in the United States in 2016 [2].

While a study in Canada found that fatigue is involved in $20 \%$ of fatal accidents [3], fatigue is also involved in $20 \%$ of commercial transport crashes in the EU [4]. To reduce the number of road accidents by $10-20 \%$, it is critical to design systems that monitor the driver's sleepiness and then warn the driver [5]. Traditionally, drowsiness detection and monitoring devices use a night vision camera to produce photographs of drivers in real time to track their condition. After that, the videos are processed and the individual frames are removed. The images are then used to either detect the face and eyes, or to construct a classification model, which is then used to detect drowsiness as shown in "Fig. 1".A noisy environment, such as poor lighting, motion blur, or magnetic interference, can cause noise to be added to the

\footnotetext{
*Corresponding Author: marwamm1987@gmail.com
} 
captured images, making face detection and extracting features from images a difficult task, particularly for real-time systems [6]. Existing systems presume that the data collected is comprised of high-resolution facial images; however, this assumption cannot be applied in real-time situations [7].
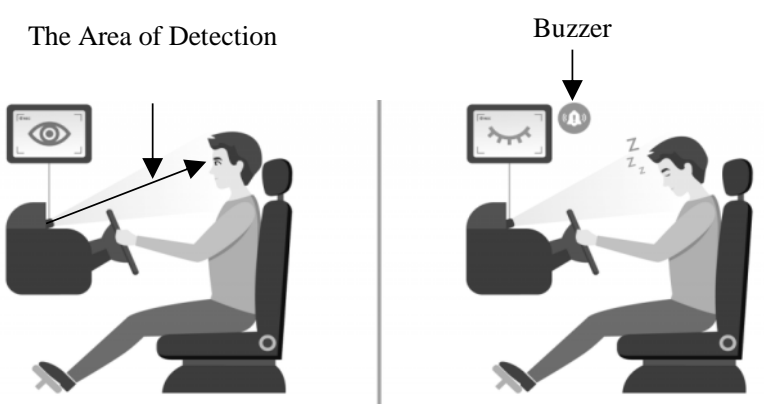

Figure 1. Principle of drowsiness detection system

Shan et al. [7] propose a system for highdiscriminative facial recognition, that combines face form and grey-level features. To evaluate the system performance they use CAS-PEALR1 dataset, which contains images of 1040 subjects captured under several conditions like variable lighting, expression and accessories. To test the robustness of the system the authors use salt and pepper noise and Gaussian noise. The system is stable Even in the presence of challenging factors such as varying lighting, noise, and partial occlusion, which significantly reduce the accuracy of a facial recognition system.

Mohammed et al. [8] propose robustness driver drowsiness detection system. The authors use Histogram of Oriented Gradients (HOG) and linear classifier, to detect face and extract features from the image, after that they measure EAR value of the eyes to detect if the driver is drowsy or not. They add to the video different random noise values, from the Gaussian distribution, to test the robustness of the system against noise. The percentage of probability of detecting landmarks decreases when the noise is increased, it become $83 \%$ when the noise level is $30 \mathrm{~dB}$, this percentage decreases to zero when the noise level is more than $130 \mathrm{~dB}$.

$\mathrm{Hu}$ et al. [9] propose abnormal driver detection system based on behavior data. The General Motors Corporation's instrumented vehicles collect Driving Behavior Data (DBD). The data take into account a variety of traffic conditions, vehicle types, and other factors, this data are unlabeled. Unsupervised pretraining with stacks of autoencoders, followed by finetuning with an Error Propagation algorithm, is a powerful method for using unlabeled information. To extract the features that characterize driving behavior and create a deep network for Abnormal Driving Detection (ADD), the wellknown deep learning model SdsAEs is used. The autoencoders can learn more robust features by adding noise, resulting in a qualified network with less interference for ADD. To avoid overfitting, a dropout technology is also used.

Sajjad et al. [6] propose facial recognition technique. The authors use three datasets JAFFE [10], MMI [11] and CK+ [12], they apply different density of salt and pepper noise to the input image to test the performance of their technique. They conclude that the accuracy decreases when the noise density is increased.

Hashemi et al. [13] propose a real-time driver drowsiness detection system using Convolutional Neural Networks (CNN). They apply three neural networks; Fully Designed Neural Network (FD-NN), Transfer Learning network (TL-VGG16), and Transfer Learning network (TL-VGG19). The Viola and Jones algorithm is used for head detection, and regression. Tree machine learning method is trained by iBUG $300-\mathrm{W}$ face landmark dataset [14] and used to detect facial landmark. The authors use ZJU dataset [15], the dataset is collected from 4 different subjects include 4,157 
images (2100 open and 2057 close images) captured by a web camera. The result shows that the accuracy of the (FD-NN) is $98.15 \%$ in different conditions.

\section{Methods}

\subsection{Proposed Dataset}

Our proposed dataset contains 1300 images, 650 images include images for drowsy faces and another 650 images for non-drowsy faces which were collected from five manually captured videos for different subjects and different camera positions .

In this work 1000 images were used during the training and validation processes where (800 images) from the total dataset were used for the training process and another (200 images) were used for validation process. Where the validation runs simultaneously with the training process to prevent model overfitting. For the testing process we used the remaining (300 images) unlabeled Images for the same subjects yet these images were never used neither in training nor the validation processes.

\subsection{Proposed Algorithm}

In the proposed framework, viola-jones algorithm [16] and Multi-task Cascaded Convolutional Neural Networks (MTCNN) algorithm [17] are considered for face detection and then used histogram equalizer, to enhance the image before it enters the classification model, to detect driver drowsiness, then alert the driver. The proposed algorithm is shown in "Fig. 2", and "Fig. 3".

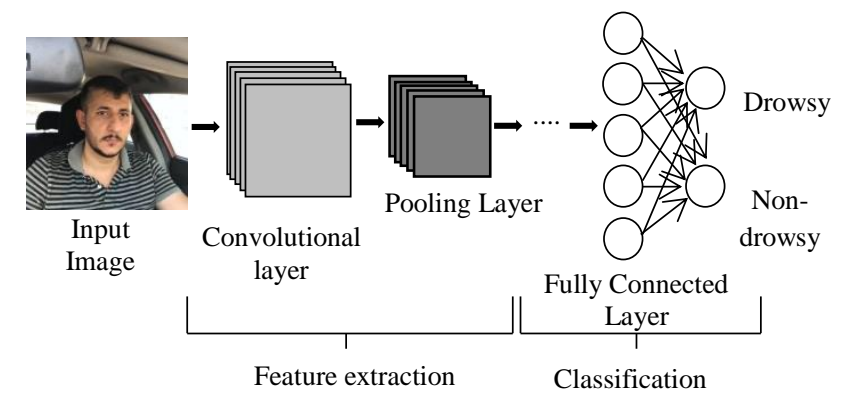

Figure 2. Proposed Algorithm Structure

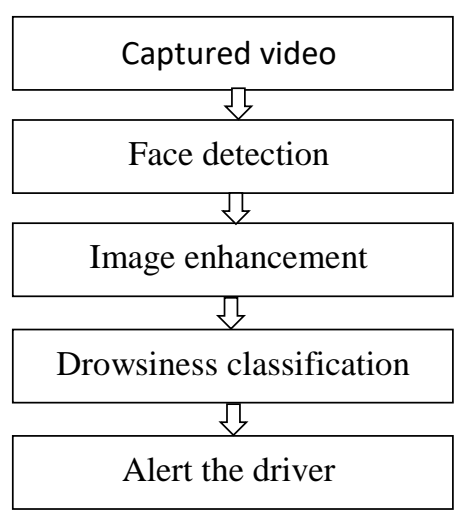

Figure 3. Proposed Algorithm Flow Chart

MobileNet V2 model is a convolutional neural networks architecture that aims to perform well on mobile devices due to its fast performance with faster processing time and low latency .

Mobile Net has 53 convolutional layers and 1 average pooling layer. MobileNet V2 model is considered to train the dataset, to detect driver drowsiness in real-time with high accuracy [18] .

The input images are set to be rotated randomly between - 90 and 90 degrees, scaled randomly, flipped randomly around the $\mathrm{x}$ and $\mathrm{y}$ axis, and resized to the resolutions [224, 224, 3] to match the Mobile Net input layer. In the proposed model the following parameters are considered, the initialize learning rate is 0.0001 , validation frequency is 5 , minimum batch size is 11 , and the hardware resources is GPU (Nvidia Georce 1050ti 4 GB). 


\subsection{MSE}

MSE is defined as a sum of square of the error between two images. MSE can be calculated using the following formula:

$M S E=\frac{1}{M N} \sum_{i=1}^{M} \sum_{j=1}^{N}|f(i, j)-g(i, j)|^{2}$

Where, $\mathrm{M}$ and $\mathrm{N}$ are the dimensions of the image in pixels, $f(i, j)$ is the corrupted image and $\mathrm{g}(\mathrm{i}, \mathrm{j})$ is the filtered image.

\subsection{PSNR}

The peak signal to noise ratio (PSNR) can be calculated using the following formula:

$P S N R=20 * \log _{10}\left(\frac{255}{\sqrt{M S E}}\right)$

\subsection{SSIM}

The SSIM is a metric for determining the degree of similarity between two pictures. Image degradation is described by SSIM as a perceived change in structural details. SSIM can be calculated using the following formula:

$S S I M=\left(\frac{(2 \mu i \mu j+C 1)(2 \sigma i j+C 2)}{(\mu 2 i+\mu 2 j+C 1)(\sigma 2 i+\sigma 2 j C 2)}\right.$

Where $\mu \mathrm{i}$ is the average of $i, \mu j$ is the average of $j$, $\sigma i$ the variance of $i, \sigma j$ the variance of $j$, $\sigma i j$ the covariance of $\mathrm{i}$ and $\mathrm{j}$ of the image $\mathrm{i} x \mathrm{j}, \mathrm{cl}=(\mathrm{k} 1$ L)2, c2= ( k2 L)2 two variables to stabilize the division with weak denominator, $\mathrm{L}$ is the dynamic range of the pixel value and $\mathrm{k} 1=0.01$ and $\mathrm{k} 2=0.03$ by default [19] [20] .

\section{Results and Discussion}

The training time for 14 epochs (1000 iteration) is 20 minutes and 45 seconds. The validation accuracy of the proposed model is $97.5 \%$ as shown in "Fig. 4", and "Fig. 5".In data augmentation a specific preprocessing step is applied to the images before training.

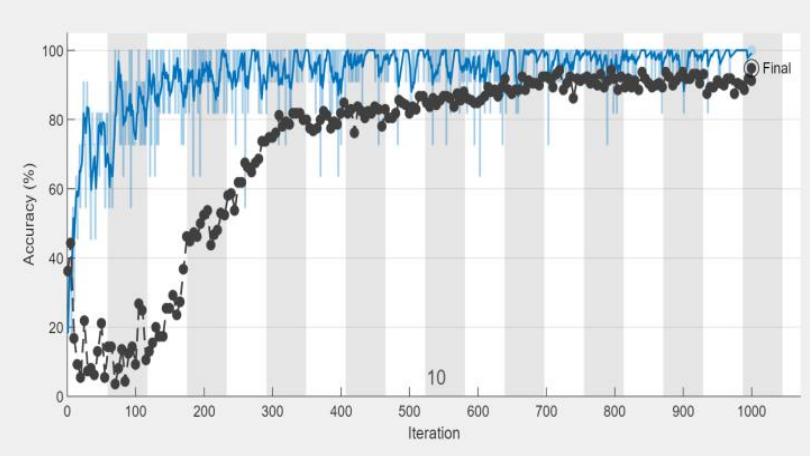

Figure 4. Training and Validation Accuracy

Training Accuracy Validation Accuracy

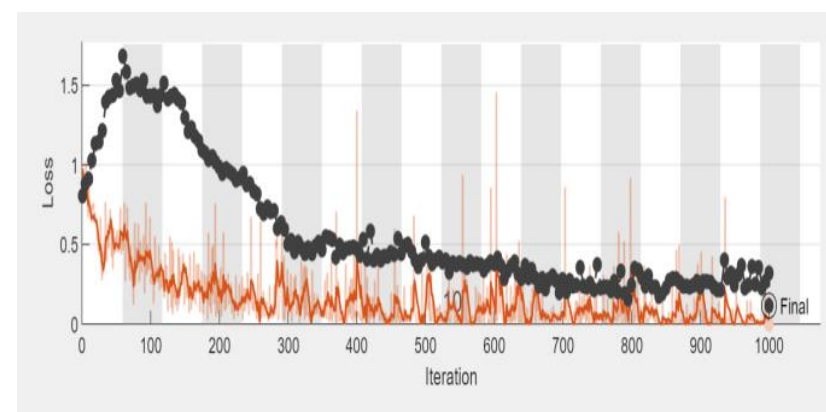

Figure 5. Training and Validation Loss

Training Loss $\square$ Validation Loss

The model is tested in two phases, in the first phase, normal captured images are used and in the second phase images with added noise are used to simulate noisy environment. In the first phase, four sets of images are tested where each set contains 100 images, with 50 drowsy faces and another 50 images for non- drowsy faces. All the images used for this testing stage are not used during the training process.

Four metrics are used to evaluate the performance of the proposed model, accuracy, f1-score, precision and recall [21] as stated in (4), (5), (6) and (7).

$$
\begin{aligned}
& \text { Accuracy }=\frac{T P+T N}{T P+T N+F P+F N} \\
& F 1_{\text {score }}=\frac{2 T P}{2 T P+F P+F N}
\end{aligned}
$$


Recall $=\frac{T P}{T P+F N}$

Precision $=\frac{T P}{T P+F P}$

Where True Positives (TP): in which nondrowsy are predicted as non-drowsy, True Negatives (TN): drowsy are predicted as drowsy, False Positives (FP): non-drowsy are predicted as drowsy, False Negatives (FN): drowsy are predicted as non-drowsy. The first phase results are shown in "Table 1".

Table 1. Test Results in Non-noisy environment

\begin{tabular}{cccccc}
\hline $\begin{array}{c}\text { Image } \\
\text { set }\end{array}$ & $\begin{array}{c}\text { No. of } \\
\text { images }\end{array}$ & Accuracy & $\begin{array}{c}\text { F1 } \\
\text { score }\end{array}$ & Recall & $\begin{array}{c}\text { Precision } \\
(\%)\end{array}$ \\
\hline S1 & $50 / 50$ & 96 & 96.15 & 92.59 & 100 \\
S2 & $50 / 50$ & 92 & 91.30 & 100 & 84 \\
S3 & $50 / 50$ & 91 & 90.53 & 95.56 & 86 \\
\hline
\end{tabular}

In the second phase the performance of the model is tested with noisy environment images, where three common types of noise are added, salt and pepper, Gaussian, and speckle noise, then we apply [3 by 3] median filter to remove the noise from the image. The filtering methods' results are measured in terms of metrics like Peak Signal to Noise Ratio (PSNR), Mean Square Error (MSE), and Structural Similarity Index Measure (SSIM).

Noise reduction is a common pre-processing phase used to enhance the results of subsequent processing (for example, edge detection on an image). In digital image processing, median filtering is very common because it works well in some situations. The median filter is a nonlinear filter used to reduce various types of noise. Finding the median value around the window, and then replacing each entry in the window, with the pixel's median value is how the median filtering is achieved. If the window contains an odd number of entries, the median is easy to calculate. It is simply the middle value after all the entries are numerically sorted. However, there are many medians for an even number of entries [20].

\section{A. Salt and Pepper Noise (Impulse Noise)}

Salt and Pepper noise is a common type of noise in images, that happens due to a camera sensor loss, software failure, or hardware failure during image acquisition or transmission. just a fraction of all image pixels is distorted in the Salt and Pepper noise model, while the rest are noisefree. A typical Salt and Pepper noise value can be either (zero) or (255). Pepper noise has a typical intensity value of around 0 and salt noise has a typical intensity value of around 255. In addition, pixels that are not affected are unaffected [20]. Where $\mathrm{P}(\mathrm{X})=0$ for pepper noise, and $\mathrm{P}(\mathrm{X})=255$ for salt noise. The effect of the salt and pepper noise on the images and applying median filter is shown in "Fig. 6". The classification results of the model after adding salt and pepper noise and applying median filter are shown in "Table 3".

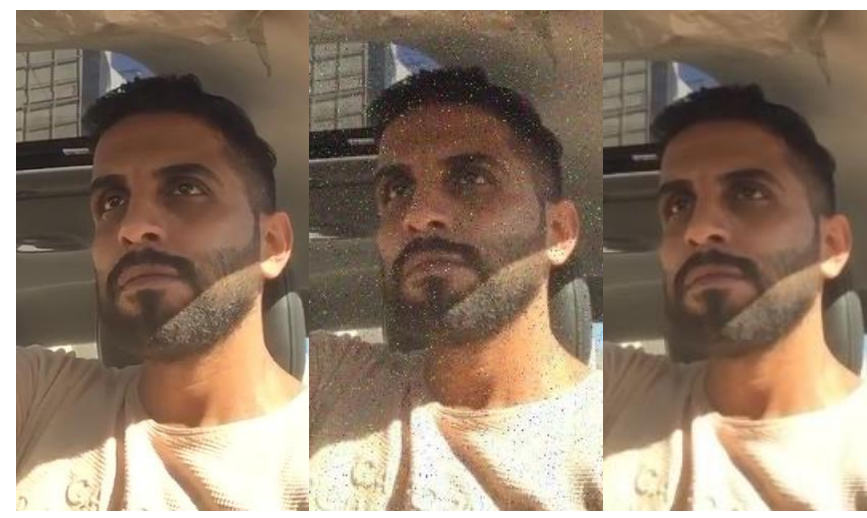

Original Image Noisy Image $\quad \begin{gathered}\text { Denoised } \\ \text { Image }\end{gathered}$

Figure 6. Testing Model with Salt and Pepper Noise

\section{B. Gaussian Noise (Additive Noise)}

Gaussian noise is generated by electronic circuit noise, sensor noise caused by high temperature, and sensor noise caused by poor brighteness. It 
is a type of observable noise, in which the noise's sufficiency is determined by Gaussian dissemination. The probability density function of the normal distribution yields Gaussian noise [22]. The effect of the Gaussian noise on the images and applying median filter is shown in "Fig. 7". The classification results of the model after adding Gaussian noise and applying median filter are shown in "Table 3".

\section{Speckle Noise}

Speckle noise is a granular noise that occurs naturally in active radar and Synthetic Aperture Radar (SAR) images and degrades their accuracy. In traditional radar, speckle noise is caused by random fluctuations, in the return signal from an object, which is not larger than a single image-processing element.

It raises the local area's mean grey level. In SAR, speckle noise is more serious, making image interpretation more difficult. Coherent processing of backscattered signals from multiple dispersed targets is the cause [23].

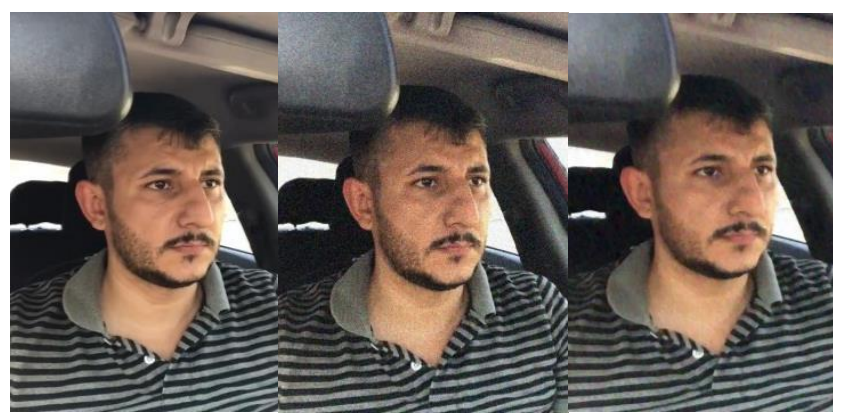

Original Image Noisy Image Denoised Image

Figure 7. Testing the Model with Gaussian Noise

The effect of the Speckle noise on the images and applying median filter is shown in "Fig. 8". The classification results of the model after adding Speckle noise and applying median filter are shown in "Table 3".

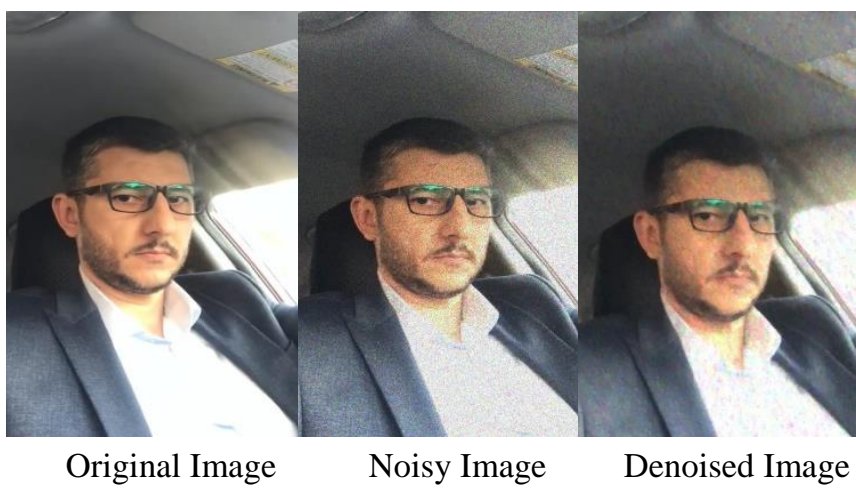

Figure 8. Testing the Model with Speckle Noise

The performance of the median filter with various types of noise is measured using the metrics PSNR, MSE, and SSIM as shown in "Table 2".

Table 2. Results of Median Filter performance with different types of noise

\begin{tabular}{llll}
\hline Subject & PSNR & MSE & SSIM \\
\hline $\begin{array}{l}\text { Salt and } \\
\text { pepper (S1) }\end{array}$ & 20.97452 & 519.6191 & 0.622911 \\
$\begin{array}{l}\text { Gaussian } \\
\text { noise (S2) }\end{array}$ & 20.42972 & 589.0827 & 0.420176 \\
$\begin{array}{l}\text { Speckle } \\
\text { noise (S3) }\end{array}$ & 19.78435 & 683.7467 & 0.332797 \\
\hline
\end{tabular}

The proposed system has proven its effectiveness in producing fast and accurate outcomes, even when various types of noise are added to the image. The model is able to classify several subjects, other than the subjects that used in the training, proving its robustness and generalization. For preparation, various camera angles and subject poses are used, which result in improved classification accuracy in a variety of driving situations. A night vision camera is need for images captured during night driving.

\section{Conclusions}

In this paper the proposed system is trained using images that manually captured for different subjects and different camera positions, and all the images used in the training 
are taken while the drivers are on the road. The dataset is collected under various lighting, resolution, and posing conditions. The trained model is fed by the entire image, rather than just the eyes area, allowing the classification model to detect the drowsiness features more accurately (eye motion, head motion, and eye closure). Due to the use of images of different subjects between the training and testing, the final validation accuracy is $97.5 \%$, while the testing accuracy for $\mathrm{S} 1$ is $96 \%, \mathrm{~S} 2$ is $92 \%$, and $\mathrm{S} 3$ is $91 \%$. Several types of noise are added to the images in testing process to check the robustness of our model. When the Salt and pepper noise is added to $\mathrm{S} 1$ the accuracy decreased to $45 \%$ and when median filter is applied the average accuracy was increased to 93\%. Gaussian noise is added to $\mathrm{S} 2$, which decrease the accuracy of this set to $85 \%$ and when the median filter is applied, the accuracy raises to $91 \%$. For $\mathrm{S} 3$ the speckle noise is added, which reduce the accuracy of the set to $73 \%$ and when the median filter is used the accuracy increased to $85 \%$. This shows that the proposed system adapts to different subjects and driving conditions.

Table 3. Test Model Results

\begin{tabular}{rrrrrrrrrrrrr}
\hline \multirow{2}{*}{ Image Set } & \multicolumn{4}{c}{ Source Image } & \multicolumn{4}{c}{ Noisy Image } & \multicolumn{4}{c}{ Denoised Image } \\
& ACC & F1 & REC & PRE & ACC & F1 & REC & PRE & ACC & F1 & REC & PRE \\
\hline S1 & 96 & 96.15 & 92.59 & 100 & 45 & 51.33 & 46.03 & 58 & 93 & 93.33 & 89.09 & 98 \\
S2 & 92 & 91.30 & 100 & 84 & 85 & 86.73 & 77.78 & 98 & 91 & 90.53 & 95.56 & 86 \\
S3 & 91 & 90.53 & 95.56 & 86 & 73 & 78.40 & 65.33 & 98 & 85 & 85.71 & 81.82 & 90 \\
\hline
\end{tabular}

\section{Acknowledgements}

This work is supported by the University of Mustansiriyah, Department of electrical engineering.

\section{Conflict of interests}

The authors confirm that the publication of this research causes no conflict of interest.

\section{References}

1. WHO, Global status report on road safety 2015. World Health Organization, 2015.

2. J. S. Higgins et al., "Asleep at the Wheel: A National Compendium of Efforts to Eliminate Drowsy Driving," Sleep, vol. 40, no. 2, 2017.

3. S. C. Government of Canada and S. C. Government of Canada, "Transport Canada," Dec. 13, 2013. https://tc.canada.ca/ (accessed Oct. 16, 2020).

4. "Mobility and transport," Mobility and transport - European Commission, 2020. https://ec.europa.eu/transport/road_safety/h ome_en (accessed Oct. 16, 2020).

5. G. K. Ramaiah, "Portable Driver Monitoring System using Matlab," IJIRMPS-International Journal of Innovative Research in Engineering \& Multidisciplinary Physical Sciences, vol. 5, no. 1, 2017.

6. M. Sajjad, A. Shah, Z. Jan, S. I. Shah, S. W. Baik, and I. Mehmood, "Facial appearance and texture feature-based robust facial expression recognition framework for sentiment knowledge discovery," Cluster Computing, vol. 21, no. 1, pp. 549-567, 2018.

7. C. Shan, S. Gong, and P. W. McOwan, "Facial expression recognition based on local binary patterns: A comprehensive 
study," Image and vision Computing, vol. 27, no. 6, pp. 803-816, 2009.

8. A. Z. Mohammed, E. A. Mohammed, and A. M. Aaref, "Real-time driver awareness detection system," in IOP Conference Series: Materials Science and Engineering, 2020, vol. 745, no. 1, p. 012053.

9. J. Hu, X. Zhang, and S. Maybank, "Abnormal driving detection with normalized driving behavior data: a deep learning approach," IEEE transactions on vehicular technology, vol. 69, no. 7, pp. 6943-6951, 2020.

10. M. Lyons, M. Kamachi, and J. Gyoba, "Japanese female facial expression (JAFFE) database," 2017.

11. M. Valstar and M. Pantic, "Induced disgust, happiness and surprise: an addition to the mmi facial expression database," in Proc. 3rd Intern. Workshop on EMOTION (satellite of LREC): Corpora for Research on Emotion and Affect, 2010, p. 65.

12. P. Lucey, J. F. Cohn, T. Kanade, J. Saragih, Z. Ambadar, and I. Matthews, "The extended cohn-kanade dataset (ck+): A complete dataset for action unit and emotion-specified expression," in 2010 ieee computer society conference on computer vision and pattern recognition-workshops, 2010, pp. 94-101.

13. M. Hashemi, A. Mirrashid, and A. B. Shirazi, "CNN-based Driver Drowsiness Detection," arXiv:2001.05137 [cs, eess], Mar. 2020, Accessed: Sep. 07, 2020. [Online]. Available: http://arxiv.org/abs/2001.05137

14. " $\mathrm{i} \cdot$ bug - resources - Facial point annotations," 2020. https://ibug.doc.ic.ac.uk/resources/facialpoint-annotations/ (accessed Sep. 18, 2020).

15. G. Pan, L. Sun, Z. Wu, and S. Lao, "Eyeblink-based anti-spoofing in face recognition from a generic webcamera," in 2007 IEEE 11th International Conference on Computer Vision, 2007, pp. 1-8.

16. Y.-Q. Wang, "An analysis of the ViolaJones face detection algorithm," Image
Processing On Line, vol. 4, pp. 128-148, 2014.

17. B. Jiang, Q. Ren, F. Dai, J. Xiong, J. Yang, and G. Gui, "Multi-task Cascaded Convolutional Neural Networks for RealTime Dynamic Face Recognition Method," in International Conference in Communications, Signal Processing, and Systems, 2018, pp. 59-66.

18. M. Sandler, A. Howard, M. Zhu, A. Zhmoginov, and L.-C. Chen, "Mobilenetv2: Inverted residuals and linear bottlenecks," in Proceedings of the IEEE conference on computer vision and pattern recognition, 2018, pp. 4510-4520.

19. A. Rehman and Z. Wang, "SSIM-based non-local means image denoising," in 2011 18th IEEE International Conference on Image Processing, Sep. 2011, pp. 217-220. doi: 10.1109/ICIP.2011.6116065.

20. R. Verma and J. Ali, "A comparative study of various types of image noise and efficient noise removal techniques," International Journal of advanced research in computer science and software engineering, vol. 3, no. 10, 2013.

21. E. Beauxis-Aussalet and L. Hardman, "Visualization of confusion matrix for nonexpert users," 2014.

22. R. Rajni and A. Anutam, "Image denoising techniques-an overview," International Journal of Computer Applications, vol. 86, no. 16, pp. 13-17, 2014.

23. S. Kaur, "Noise types and various removal techniques," International Journal of Advanced Research in Electronics and Communication Engineering (IJARECE), vol. 4, no. 2, pp. 226-230, 2015. 